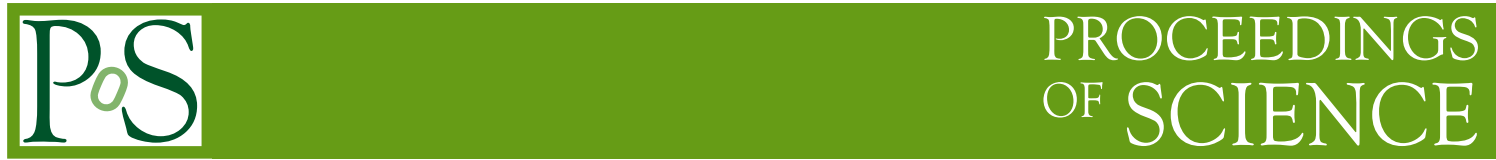

\title{
Wednesday question and answer transcripts
}

\section{Lanny Ray*}

Department of Physics, The University of Texas at Austin, Austin, Texas 78712 USA

E-mail: ray@physics.utexas.edu

This is a transcript of the questions and answers following the Wednesday presentations. The audio recordings were generally transcribed as is but were in some cases edited for clarity, where every effort was made to be faithful to the speaker or participant's intended meaning. Additions inserted during the editing process to clarify the speaker's intent are enclosed in square brackets. Additional, contributed comments made after the workshop are identified as "Note added." Unintelligible audio portions are indicated by "[???]." Participants were: Rene Bellwied, Helen Caines, Yuri Dokshitzer, Rainer Fries, Ahmed Hamed, Uli Heinz, Rudy Hwa, Jiangyong Jia, David Kettler, Che-Ming Ko, Boris Kopeliovich, Christina Markert, Denes Molnar, Guy Moore, Lanny Ray, Thorsten Renk, Lijuan Ruan, Edward Shuryak, Raimond Snellings, Mike Tannenbaum, Derek Teaney, and Tom Trainor.

Workshop on Critical Examination of RHIC Paradigms - CERP2010

April 14-17, 2010

Austin Texas USA

${ }^{*}$ Secretary. 


\section{Yuri Dokshitzer}

Edward: The LUND model has been used successfully in describing confinement and hadronization. If you use the LUND model for these data, what do you get?

Yuri: The LUND model was successfully used for some 7-10 years before the model was pushed by the LEP data which required that the model be changed.

Edward: I understand that you can use your application of perturbative QCD in its region of validity and not LUND. But if we consider $300-400 \mathrm{MeV}$ pions, how do your calculations apply to those data? If we use the LUND model with its canonical string tension will it fail?

Yuri: Yes, it fails. The few hundred $\mathrm{MeV}$ particles "remember" that they came from few $\mathrm{GeV}$ partons. The LUND model does not have that feature and will get it [yields] wrong because it has no particle multiplication mechanism which was required by the LEP data. The LEP data forced the LUND model to include perturbative particle multiplication.

Edward: But you wouldn't have to change anything about the confinement in the LUND strings, they would still break and produce hadrons.

Yuri: No.

Edward: When you say "soft" does it mean the string tension should be reduced, or not? What do you mean by "soft?"

Yuri: No, the string tension does not have to be reduced. By "soft" I mean that the strings must be easily broken. This doesn't mean we have to change the confinement parameters in the LUND model.

Tom: I want to ask you to comment about the blanching process à la Kogut-Susskind, the statement that there is no recombination in vacuum processes, and what are the modifications in large spacetime volumes?

Yuri: Regarding blanching, this is a consequence of causality and quantum mechanics. There cannot be cross-talk between the partons produced in $\mathrm{e}^{+}-\mathrm{e}^{-}$collisions. In the 80's people believed that Pomeron exchanges would enable cross-talk between the partons. In p-p collisions maybe this is not the case because the partons do not emerge from a point as in $\mathrm{e}^{+}-\mathrm{e}^{-}$.

Tom: What about quantum non-locality?

Yuri: This was what was driving Richard Feynman when he introduced partons because he did not want the quarks to fly apart as fractional charges. He introduced a picture in which something would happen after $1 \mathrm{fm} / \mathrm{c}$ and this something is local blanching. This blanching, or hadonization happens continuously. It is a long duration process. It starts at $1 \mathrm{fm} / \mathrm{c}$ and ends at $E \mathrm{fm} / \mathrm{c}$ where $E$ is the $\sqrt{s}$.

Tom: Now what happens in heavy ions?

Yuri: A lot of things happen in heavy ions [laughter]. I will show three undigested phenomena. One is how the LPM effect in heavy ion collisions contradicts participant scaling [not understandable?]. The second is about the role of color in multiple collisions. If you consider high energy collisions as the result of one gluon exchange, then the graphs depend only on the color of the exchange, and the answer only depends on how much color is exchanged. In naive pion scattering the pion 
is originally colorless, but after gluon exchange it becomes colored, and on successive collisions it remains colored, forming two color chains, one the color image of the other. From the color point of view the state is initially a color-singlet and finally it is a color-octet. The point is that whether there is one or many exchanges the final state will still be an octet, which means that successive collisions of the particle do not produce additional color. The third comment is about confinement and recombination. When we look at heavy flavor suppression in quarkonia people always talk about final state interactions, which in my view is saying that fragmentation is not independent. So people talk about their models of interacting strings, color ropes, etc. But from the point of view of color, even in pA collisions, there is a new environment being formed. How does confinement respond in a strong field environment? This could have been addressed at LEP.

Edward: How do you get strong fields at LEP?

Yuri: There are some ideas using multi-jet events looking at the soft particles produced, such as the inter-jet particles which are not part of any jet but where the field strength could be enhanced. I think that the strength of the color fields within a perturbative approach can explain the suppression. Stronger fields mean the vacuum breaks earlier, earlier means shorter distances which means it is less sensitive to the mass squared dependence of the strange quark for example. I also think that the geometry of the strong color fields could come into play in explaining many of the heavy ion phenomena.

\section{Lijuan Ruan}

Mike: Is there any difference in the particle/resonance ratio between d-Au and $\mathrm{Au}-\mathrm{Au}$ ?

Lijuan: The peripheral $\mathrm{Au}-\mathrm{Au}$ is similar to the d-Au data. The d-Au data are not shown here but are in a separate reference.

Edward: Radial flow in Au-Au could be different than it is in d-Au at the same Npart, so there could be some differences.

Lijuan: But rescattering effects in more central collisions could offset the radial flow effect, leaving the observed small centrality dependences in the particle ratios.

Edward: How can the fragmentation functions be wrong. Aren't they fixed by $\mathrm{e}^{+}-\mathrm{e}^{-}$data?

Mike: Different theorists give different answers, e.g. DSS and AKK.

Lijuan: The fragmentation functions from $\mathrm{e}^{+}-\mathrm{e}^{-}$usually measure combined pairs such as $\mathrm{p}$ and $\overline{\mathrm{p}}$, whereas at RHIC we measure both $\mathrm{p}$ and $\overline{\mathrm{p}}$ separately and get an accurate $\mathrm{p} / \overline{\mathrm{p}}$ ratio. In $\mathrm{e}^{+}-\mathrm{e}^{-}$ fragmentation functions the light quark contributions are not separated.

Yuri: The quarks are not separated but the hadrons are [separated] in $\mathrm{e}^{+}-\mathrm{e}^{-}$data.

Lijuan: Yes, but not precisely. For example, AKK used separated particle and anti-particle yields but it is not precise.

Mike: Why are the particle ratios for $(+)$ and $(-)$ combined in your plots, rather than being separated? It is my experience that $\mathrm{K}^{+}$and $\mathrm{K}^{-}$are quite different.

Lijuan: Yes, they are different. The charges were combined here in order to compare charged kaon and $\mathrm{K}_{s}^{0}$ yields. 
Edward: Are the $\mathrm{K} / \pi$ ratios to $14 \mathrm{GeV} / \mathrm{c}$ consistent with standared fragmentation functions, $\mathrm{AKK}$ etc.?

Lijuan: Both AKK and DSS global fits are about a factor of two too large.

Yuri: At these higher momenta the kaons are affected by the particle multiplication mechanism.

Edward: Do we see any puzzles at $14 \mathrm{GeV} / \mathrm{c}$ where none were expected? Do the usual fragmentation mechanisms work there?

Mike: Referring to the AKK fragmentation model, why do theorists claim that protons come from gluon jets rather than quark jets? It seems much harder for a gluon to make proton - antiproton pairs than for quarks.

Yuri: There was an experimental observation that the proton spectra from gluon jets are steeper, thus there is a difference.

Boris: Did you compare the RHIC K/ $\pi$ ratios to Tevatron data?

Lijuan: I did not compare with Tevatron data but STAR and especially PHENIX compared $x_{t^{-}}$ scaling data with CDF pion and proton data. Experimentally the data are consistent above $p_{t}=2$ $\mathrm{GeV} / \mathrm{c}$. For proton fragmentation both quark and gluon contributions are probably comparable, but $\overline{\mathrm{p}}$ production is probably dominated by gluon contributions.

Boris: Do the fragmentation functions show the same contributions?

Yuri: Gluon fragmentation produces some ratio of protons to mesons which is large. Additional quarks spoil it.

Edward: But the proton to meson ratios will depend on the system and are not the same as in $\mathrm{e}^{+}-\mathrm{e}^{-}$, ie. not universal.

Thorsten: This all tells us that the fragmentation functions are not as well constrained by experiment as we would like.

Edward: If the error bars are included on the AKK and DSS fragmentation models will everything be consistent?

Thorsten: Only in AKK is proton fragmentation predominantly from gluons. There was a tremendous change in the parametrization in response to new data which shows that the fragmentation functions were not well constrained experimentally in the first place.

Edward: If the error bars are included is everything obscured, and the data and models are all within the error bars and there is then no puzzle and we should not worry?

Yuri: Let me comment about the obscurity issue. Here [in p-p] it is unfair to call this process fragmentation [as in $\mathrm{e}^{+}-\mathrm{e}^{-}$] because the environment is different from in $\mathrm{e}^{+}-\mathrm{e}^{-}$. Here there is a trigger which requires that there is a leading gluon which carries $99 \%$ of the momentum. These are strongly biased measurements.

Edward: But assuming factorization the $\mathrm{K} / \pi$ ratio should be predicted in $\mathrm{p}-\mathrm{p}$ collisions.

Yuri: Yes, ratios are better.

Guy: The $\mathrm{K} / \pi$ ratio is not as reliable for testing fragmentation functions in $\mathrm{p}-\mathrm{p}$ as you might think. If the data are from $\mathrm{e}^{+}-\mathrm{e}^{-}$then you would expect a lot of $\mathrm{s}+\overline{\mathrm{s}}$ pairs, but in $\mathrm{p}-\mathrm{p}$ I doubt that any of 
these kaons are from jets with a leading strange particle.

Yuri: Are the $\mathrm{K}^{+}$yields larger than $\mathrm{K}^{-}$?

Lijuan: Yes. The KKP fragmentation functions are about a factor 10 lower than the data. AKK improves on this prediction. The fragmentation functions are not well constrained at all.

Mike: Why doesn't everyone understand that AKK is wrong and stop using it?

Tom: It is important to realize that AKK did a global fit to combined data from $\mathrm{e}^{+}-\mathrm{e}^{-}$and $\mathrm{p}-\mathrm{p}$ and DIS and that if fragmentation is not universal then their resulting functions are being pulled to an intermediate position and will not describe either one accurately.

Mike: It may not be universality. In $\mathrm{e}^{+}-\mathrm{e}^{-}$measurements the energy is at the $\mathrm{Z}$ mass.

Tom: In p-p [collisions] if mid-rapidity jets result from hard-Pomeron exchange then that is a four jet process [two jets near mid-rapdity connected by momentum transfer but color connected to two jets at large rapidity], and the fragmentation is very different from that in $\mathrm{e}^{+}-\mathrm{e}^{-}$.

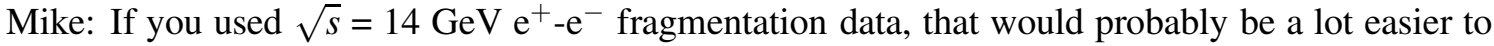
compare to $\mathrm{p}-\mathrm{p}$ data rather than the high energy $\mathrm{e}^{+} \mathrm{e}^{-}$data at the $\mathrm{Z}$ mass.

Note added (Tom): A complete parametrization of $\mathrm{e}^{+}-\mathrm{e}^{-}$fragmentation functions for light quarks and gluons fragmenting to unidentified hadrons and for dijet energies from 10 to $200 \mathrm{GeV}$ is available in Phys. Rev. D 74, 034012 (2006). Related fragment distributions comparable to p-p collisions are described in Phys. Rev. C 80, 044901 (2009).

Boris: In $\mathrm{e}^{+}-\mathrm{e}^{-}$they have straightforward fragmentation functions, but in $\mathrm{p}-\mathrm{p}$ we measure fragment distributions that are a convolution of fragmentation functions with parton distribution function, with a strong bias in the observed kaon, pion distributions. So what do we conclude?

Lijuan: In AKK they use CTEQ parton distribution functions, calculate the parton distributions, and use the fragmentation functions to get the particle spectra [spectrum hard components]. The KKP results were not included because they are a factor of 10 below the data. Even with the theoretical uncertainty included I do not think KKP can reproduce the data. The difference between AKK and KKP is that the AKK group used more recent data including light quark tagged data. There is improvement but not enough to describe the data.

Mike: Why is the $\eta / \pi$ ratio flat at high $p_{t}$ ? And for $\mathrm{R}_{A A}$ also? I am not saying this is a puzzle, but it is an observation.

Jiangyong: Given the complexity of the $\mathrm{R}_{A A}$ data for many identified particles it seems clear that the simple picture of jet quenching as energy loss in the medium followed by fragmentation outside the medium is too simple. Because of flavor changing processes this is much more complicated. You commented that anti-protons are more likely from gluons than are protons. Do you have separate $\mathrm{R}_{A A} \mathrm{~s}$ for $\mathrm{p}$ and $\overline{\mathrm{p}}$ ?

Lijuan: Yes we do and we do not see a difference in $\mathrm{R}_{A A}$ between $\overline{\mathrm{p}}$ and protons.

Thorsten: It is rather obvious that heavier hadrons like the proton cannot hadronize outside the medium based on the energy over mass squared time scale which puts their hadronization inside the medium. The simple picture of energy loss in the medium with fragmentation outside cannot 
apply for heavy particles. For heavier hadrons their hadronization is dragged inside the medium and the process becomes quite complicated.

Jiangyong: Does that mean the jet quenching mechanisms are best for pions?

Thorsten: For light hadrons at high $p_{t}$ the calculations are good, but if you go to heavy hadrons at low $p_{t}$ the calculations are not reliable.

Denes: But these data were for $14 \mathrm{GeV} / \mathrm{c}$ protons so they should form outside the medium and the calculations should be applicable.

Edward: Are the calculations all right for $\mathrm{K} / \pi$ and $\eta / \pi$ ratios at $14 \mathrm{GeV} / \mathrm{c}$ ?

Mike: If heavier hadrons like protons form in the medium why aren't they immediately dissolved like the $\mathrm{J} / \Psi$ is supposed to be?

Edward: If the $\mathrm{J} / \Psi$ moves with a large momentum then it is not dissolved.

Yuri: Coming back to the question of fragmentation, for ten years we have been hearing the same message at conferences, that QCD is dead because there are so many more sea[?]-quarks at the Tevatron. But the problem is due to non-trivial errors in the fragmentation functions. The fits have to describe not just the shape but also the tiny region where $\xi$ [-log of momentum fraction?] is close to 1 .

\section{Tom Trainor}

Yuri: What is the parameter $v$ ? Is it the same as used in $\mathrm{p}+\mathrm{A}$ collisions?

Tom: Yes, in the Glauber model $v$ is the number of binary collisions $N_{b i n}$ per participant pair $\mathrm{N}_{\text {part }} / 2$, and can be interpreted as the average path length of a nucleon measured in number of nucleons. $v$ varies from 1 to 6 in Au-Au collisions.

Edward: Is your assumption that there are only hard processes and nothing else?

Tom: In p-p collisions yes. The hypothesis being tested is that soft Pomeron exchange gives the participant number scaling element of the model, such that no matter what the centrality of the A-A collision nucleons interact with nucleons to produce the PDF of the nucleon. In addition there are large-angle scatterings. What I am calling 'hard' processes are large-cattering-angle processes [at any parton energy scale].

Edward: So your hypothesis is that Au-Au is a superposition of p-p collisions?

Tom: Yes, in the plots this limit is referred to as Glauber linear superposition or GLS.

Note added (Tom): The GLS is a reference (null hypothesis) for A-A collisions. The data deviate from the reference in more-central collisions, and the deviations represent what is new in A-A collisions.

Rene: The parton spectrum must depend on collision energy.

Tom: Here I am using the Cooper et al. spectrum for $200 \mathrm{GeV}$ but have no comment about the $\sqrt{s}$ dependence of the parton spectrum. 
Edward: The soft component is much larger than the hard component in Au-Au so when the soft part is subtracted from data the resulting differences with centrality could all be within the error bars.

Tom: Yes, the relative ratio is a factor ten and you could say that. But the difference curves do show the centrality dependence relative to a fixed function.

Note added (Tom): Although the soft component dominates spectra at smaller $p_{t}$ it is still possible to obtain quite accurate estimates of the hard component down to $0.5 \mathrm{GeV} / \mathrm{c}$ or even lower by a limit approach, as first described for p-p collisions in Phys. Rev. D 74, 032006 (2006) and extended to A-A collisions in Int. J. Mod. Phys. E 17, 1499 (2008).

Jiangyong: The soft component that is subtracted is the same at all centralities, right?

Tom: That's right, I assume that. So this is open for debate. But what this shows is the fine structure of the spectra which has never been done before. This is a differential study.

Helen: You said the integral of the soft and hard spectrum components is maximally different in [central] Au-Au [per participant pair] but is the energy the same?

Tom: No, the Borghini and Wiedemann model redistributes the energy. This result is consistent with no energy being lost from a jet.

Helen: But these curves are data, not the Borghini and Wiedemann model. So do the data conserve energy?

Tom: Yes, up to a point. But at low $p_{t}$ the errors in the subtraction are too large to say.

Ahmed: Has anyone done this kind of analysis for the d-Au data?

Tom: No, but I note that the centrality determination in d-Au is tricky and what we see in $\mathrm{Au}-\mathrm{Au}$ is a sharp transition in the centrality evolution. I would come back to d-Au later after understanding $\mathrm{Au}-\mathrm{Au}$.

Ahmed: What do you think is the underlying physics of the transition you see around $v=3$ ?

Tom: I can only say phenomenologically that this transition occurs where the number of jets in a $\mathrm{Au}-\mathrm{Au}$ collision rises above one. The probability of jets in $\mathrm{p}-\mathrm{p}$ is 0.01 roughly. The number of jets in central $\mathrm{Au}-\mathrm{Au}$ is of order 70 minijets or minimum biased jets. Somewhere in between the number of jets passes through one and that is where the action starts [the sharp transition in jet characteristics].

Ahmed: Have you examined $\mathrm{R}_{A A}$ all the way down to the soft component?

Tom: No, I would not use $\mathrm{R}_{A A}$ at all because below $6 \mathrm{GeV} / \mathrm{c} \mathrm{R}_{A A}$ is increasingly dominated by the soft component and so the sensitivity to fragmentation is obliterated. Below $4 \mathrm{GeV} / \mathrm{c} \mathrm{R}_{A A}$ is dominated by the soft component in the numerator and denominator and is proportional to $1 / \mathrm{v}$. There is no physics in $\mathrm{R}_{A A}$ at these lower momenta. It is totally misleading.

Thorsten: What you would call energy loss I would call perturbative energy redistribution within the jet as described by Borghini and Wiedemann and any of the new shower Monte Carlo models. But all these mechanisms require that there be some interaction with a medium, which I guess you would call the soft component. I do not know, but you need something for the parton to interact with. 
Tom: Yes, I agree with all this.

Note added (Tom): Experimental data are consistent with the soft component (from projectile nucleon diffractive dissociation) not interacting with the jet system. Thus, what jets interact with to be modified may be other jets, or a "medium" whose origin is not clear.

Thorsten: My crucial comment is that you can look at gamma-hadron correlations and for the 180 degree hadrons within a cone measure the distribution of correlated hadrons as a function of $\xi[\xi=$ $\ln (1 / x)$ where $x$ is the momentum fraction of the produced hadron]. Such data can be compared with shower Monte Carlo models or Borghini and Wiedemann. However, these predictions do not agree with the data. I will present this in my talk. I take this to mean that perturbative redistribution within a jet does not happen at RHIC. All redistribution models I have seen predict a very similar distortion of the fragmentation function.

Tom: We adopted the Borghini and Wiedemann model only as a proof of principle. My interest is in showing that you can make these calculations all the way down to zero fragment momentum. Other modification mechanisms could be used for the same purpose. I am not claiming that Borghini and Wiedemann is correct. I am only saying that if you had something like their model, this is how the distortion would look and would compare to the data.

Thorsten: Have you ever looked at this type of gamma-hadron correlation?

Edward: You are assuming the Glauber binary scaling for the hard component and the new element is the Borghini and Wiedemann modification and then you get $R_{A A}$ at high $p_{t}$, which is more or less the standard analysis. The non-standard aspect of your analysis is that you follow these fragments to small $p_{t}$, but these soft fragments hide below the other soft particles.

Tom: I am saying that they do not hide, that they can be separated.

Edward: But the soft fragments are an order of magnitude less than the total number of soft particles so they are difficult to find.

Tom: There is a paper on this separation with a discussion of systematic errors.

Edward: Is your statement that you can find these few percent of particles that ignore the explosion and are described perturbatively?

Tom: They do not completely ignore the explosion. There is a dramatic change in central $\mathrm{Au}-\mathrm{Au}$, they [low $p_{t}$ fragments] increase by a factor of twenty.

Edward: But that is a trivial multiplication by hard scaling. The issue is that after they are produced they ignore all the other soft particles.

Note added (Tom): The factor twenty increase is relative to "hard scaling" (binary collision scaling).

Tom: A parton in central Au-Au that is going to turn into a jet is very aware of its surroundings. But whether that is a bulk medium or other colored objects from scattered partons, I do not know, but it is very different in Au-Au.

Edward: So what is your statement? You say there is this hard component which is the fragmentation of hard jets which is already in p-p but is very small, and is a small part of the total yield in $\mathrm{Au}-\mathrm{Au}$. 
Note added (Tom): "Hard jets" in this case includes jets (large-angle scattered partons) down to 3 $\mathrm{GeV}$.

Tom: In p-p it is small but in Au-Au it is one-third of the final state.

Edward: I don't see that from the soft background, how it can be 1/3.

Denes: This issue is really the crux of this analysis and the interpretation.

Tom: The jet correlations are telling you that they are $1 / 3$ of the final state.

Note added (Tom): Relative to p-p collisions the jet abundance per participant pair in central AuAu increases by a factor $v=6$ from geometry and by $60 \%$ due to an inferred dijet cross-section increase in central Au-Au vs p-p. The mean jet fragment multiplicity increases by a factor 3, as inferred from jet angular correlations. The overall increase over the $1 \%$ jet contribution in NSD p-p collisions is then a factor 30 in central Au-Au collisions. Approximately 1/3 of the final state is observed to be jet-correlated hadrons.

Mike: Can you make predictions for the LHC?

Tom: I have not yet, but I have given you the tools to do so.

\section{Rudy Hwa}

Guy: How does your model explain the very long range in $\eta$ [pseudorapidity] of the ridge?

Rudy: Here I am only considering mid-rapidity but the ridge will be discussed later by Charles Chiu.

Thorsten: The characteristic feature of the ridge is that it is a long range $\eta$ correlation, not that it is an enhancement.

Rudy: The $\eta$ elongation is a different problem than what I am discussing here. [It will be explained in a paper to come. See comments in Saturday's discussion session.]

Che-Ming: In the calculation of your path length variable you neglected the expansion of the medium.

Rudy: In order to include expansion I would have to include additional models. Here I am only considering initial conditions and assume those are what will determine what happens. But the time it takes for recombination to occur is short for semi-hard partons not too far from the surface, so the medium doesn't expand that much.

Rene: Can I learn anything from the dynamical path length? For instance, what does the cut-off at 0.6 mean and why doesn't it go further out? Is 1.0 the full normalized path length through the nucleus? Your dynamical path length scaling suggests that $\mathrm{R}_{A A}$ will go below 0.2 for larger path lengths. What governs the dynamical path length?

Rudy: The maximum geometrical value for the pathlength is $2 \mathrm{R}$ which is for central collisions traversing the entire system. But after averaging over density and azimuth, $\bar{\xi}$ comes down to 0.6 [as a dimensionless measure of dynamical path length]. That is where $\mathrm{R}_{A A}$ reaches its minimum of 0.2. [Rudy: I can't explain simply the significance of $\bar{\xi}$ being less than 0.6.]

Mike: What is your prediction for $\mathrm{v}_{2}$ at the LHC? 
Rudy: I do not have such a prediction at this time.

Mike: What is the difference between a "valon" and a constituent quark?

Rudy: Constituent quarks are what are used in bound state problems. But what we need are momentum fractions in the infinite momentum frame. There is much more relevant structure than in the bound state problem. But the valon is the same as a constituent quark in the rest frame in the context of the bound state problem.

Mike: So it would be nice if you would apply this model to spin.

Rudy: Yes, but I have not talked about spin in the valon model.

Mike: You said you can explain the Cronin effect in your model. Can you please explain?

Rudy: The Cronin effect is usually regarded as an initial state effect in which the transverse momentum is due to initial scattering. If that is the case then how do we explain the fact that proton yields are increased much more relative to pions? This can be explained as recombination in the final state.

Thorsten: You made a point that your's and Tom's models are very similar, but I see them as being orthogonal. What you assume is that the energy lost by a hard parton is thermalized. That is why you have thermal-thermal and thermal-shower recombinations. So you have a weird state which is thermal yet doesn't expand like hydro models do. Tom's model is that of a perturbative redistribution of energy within a shower which is not thermal at all but involves completely different dynamics. So both models have energy conservation and both have 2 or 3 components for soft and hard momentum but otherwise I do not see any common ground between the two.

Rudy: I suggested the common ground in words, but the details have to be worked out.

Thorsten: Well it is not so much a detail as whether the medium is thermalized or not.

Edward: Tom proposes that two components are sufficient, whereas you would have the shower and thermal particles coalesce. I think Tom would object to that. I would say Tom is right in that the high $p_{t}$ particles move at the speed of light while the soft-thermal particles move at the speed of hydro which is small. How does recombination work when the shower partons and thermal partons are at different speeds and spatial locations after some time when they form hadrons?

Rudy: I have not included time evolution since that would require additional models, since hadronization occurs at the end of the evolution.

Edward: But at the end the hard particle fragments are several fermis away from the soft-thermal particles.

Rudy: The hard parton breaks into shower partons which are at lower momentum similar to the thermal partons.

Edward: Yes, they may have similar momenta but they are spatially separated after some time.

Rudy: Not necessarily. There is a collection of all possible soft and hard partons to consider. [Rudy: It is like in the parton model of a proton. There are high-x and low-x partons. How can they be in the same spatial volume of the proton? The sea quarks are due to local excitation from the vacuum. The PDF takes care of that. So also does the recombination function take care of coalescing partons that have different momenta and apparently different spatial positions.] 
Lijuan: Can you clarify whether your results contradict hydro or just say that hydro is not necessary?

Rudy: With increasing time the thermal partons will produce expansion and thermalization, but in the shower description the azimuth asymmetry is not driven by immediate pressure gradient differences as in hydro. The semi-hard partons are going in different directions and they can drive the asymmetry. That is the main point. The geometry affects the probability of the semi-hard partons going in one direction or another.

\section{Raimond Snellings}

Yuri: Why does one need to talk about pressure at all? Because it is sufficient to have just some geometry and absorption.

Raimond: Yes, or some interactions, that is correct.

Edward: People started with pQCD. Miklos [Gyulassy] started with the LUND model and what he found is that there is a significant asymmetry [momentum or number???] which is larger in this direction [perpendicular to the reaction plane] than it that direction [in the reaction plane]. Why? Because when the particles multiply they multiply more in one direction than the other. So in principle you can get an asymmetry but it had the wrong sign and the wrong magnitude.

Raimond: That is true. I am talking about pressure here because I am taking it to the extreme in the hydro limit. It is easy to imagine but it is certainly not necessary and I am not sure if that is what is required.

Tom: To be clear [in the cumulant expansion] you did not get rid of non-flow; you got rid of an element of non-flow.

Raimond: I got rid of two-particle correlations.

Tom: Right, by hypothesis.

Raimond: What do you mean "by hypothesis"?

Tom: You are assuming this expansion describes reality.

Raimond: No, this has nothing to do with reality; this is just mathematics. I am not saying what these components are. These are just definitions and mathematics.

Denes: Can you put a rigorous upper bound on these $1 / \mathrm{M}$ [cluster multiplicity] limits? Is it really 1/M, 2/M, 5/M, 10/M, 100/M...?

Raimond: If I make them [the cluster particles] in exactly the same [direction?] then they are correlated of order one, but if there is an opening angle then that is already different, and then you have the rest of the particles.

Denes: Of course you know the 1/M but if you do not know the coefficients then all these 7\%, $2 \%$ are up for grabs. There must be a rigorous upper bound using your arguments.

Raimond: The correlations between the particles cannot be bigger than one. But then the $\mathrm{M}$ is not really well known.

Denes: I understand, it is rigorously less than or equal to $1 / \mathrm{M}$. 
Derek: I have a question about the two-particle correlations. You say [reliable measures must be much greater than 7\%] and you guys use two-particle correlations and you get an estimate of $\mathrm{v}_{2}$ but it is not $100 \%$ off. So there must be some other factor in these $1 / \mathrm{M}$ limits.

Raimond: The correlations may not be as strong and they are not all in pairs. So this estimate is already rather extreme.

Tom: It would be better to say, wouldn't it, that it is proportional to that $[1 / \mathrm{M}]$, which is giving you the functional form and you should not infer anything about the absolute magnitude.

Raimond: Yes

Derek: Would you trust the two-particle method to $30 \%$ ?

Raimond: At RHIC yes, but at the LHC I would not at this point and that for me is very important. So I would certainly do all these higher order calculations.

Tom: In your simulations you have no back-to-back elements in the simulations for non-flow?

Raimond: If it would just be two particles back-to-back then that would be fine.

Tom: But you are taking each particle twice which means that you have a same-side peak but no away-side.

Raimond: But if I have a back-to-back peak it is the same. If I do more then things will break. If I make more than two-particle correlations then I certainly [not understandable?] the effect.

Tom: So these results are for a rather special case.

Raimond: Sure, the magnitude is for a special case. But if it's just two-particle correlations then these contributions are removed. That is direct from the mathematics.

Ahmed: This [ $\mathrm{v}_{2}$ agreement between cumulants and $\left.\mathrm{ZDC}\right]$ is due to the poor reaction plane resolution of the ZDC and would have a huge correction.

Raimond: That would be extremely accidental if the resolution just matches so you get the same $\left[\mathrm{v}_{2}\right]$ value out. That's really far-fetched.

Tom: So you are assuming that what is generating the $\mathrm{v}_{2}$ is point-like nucleons.

Raimond: What I am assuming is that the eccentricity coming from a Glauber model is somewhat related to the true eccentricity.

Yuri: Do you expect a thermalized medium with the densities in a $\mathrm{Cu}-\mathrm{Cu}$ collision?

Mike: It happens whether you expect it or not [laughter].

Raimond: I am not saying it is a thermalized system, but there is a system with flow in $\mathrm{Cu}-\mathrm{Cu}$. There are multiple interactions and that generates $a v_{2}$ and that $v_{2}$ scales between the two systems depending on the multiplicity.

Derek: It is half [in $\mathrm{Cu}-\mathrm{Cu}$ ], that is the important thing.

Raimond: Yes, compared to central $\mathrm{Au}-\mathrm{Au}$, but that is fine.

Tom: The back-to-back jet contribution in UrQMD is what? [none] Right, so what are you testing in that exercise [comparison of $\mathrm{v}_{2}$ cumulants to true $\mathrm{v}_{2}$ in UrQMD]? 
Raimond: Well I am testing the mechanism. I am saying that multi-particle methods measure the flow in the true reaction plane, which is true. And this thing contains fluctuations, correlations, and if you want you can embed jets and do the same exercise and you will find that the conclusion holds, unless you have only two back-to-back jets and that is all in the system.

Lijuan: Can you comment on why the non-flow effect in $\mathrm{Cu}-\mathrm{Cu}$ collisions is bigger than in $\mathrm{Au}-\mathrm{Au}$ collisions, for example, using two-particle correlations with the TPC in STAR?

Raimond: The main thing is that if the multiplicity becomes large then the non-flow becomes smaller. So if you assume the [fraction of] multiplicity associated with jets in $\mathrm{Cu}-\mathrm{Cu}$ is the same as in $\mathrm{Au}-\mathrm{Au}$, which is probably not so far off, then the correlations in $\mathrm{Au}-\mathrm{Au}$ are much more diluted because there are many more particles and jets, and then the non-flow contributions therefore become less.

Note added(Tom): That is simply because $v_{2}^{2}$ is a pair ratio. "Non-flow" specified in that context includes a factor $1 / n_{c h}$. The dominant source of "non-flow" (jets) increases absolutely with increasing A-A centrality.

Tom: Just a clarification - when you conclude collective flow you are testing up to $n=4$ only, right?

Raimond: No, certainly in STAR we did 8-particle cumulants and we did Lee-Yang Zeros.

Tom: Over what centralities did you get 8-particle cumulants? Or was it done for minbias?

Raimond: It [8-particle cumulant analysis] was done as a function of centrality which was 10-70\%.

Tom: So is this statement correct? "For some centralities you have established that at least eight particles are involved in this $\left[\mathrm{v}_{2}\right]$ phenomenon."

Raimond: That and more because the Lee-Yang Zeros tell you there are much more than eight particle correlations.

Tom: How is that the case? Why does Lee-Yang say there are more than eight?

Raimond: The Lee-Yang zeros are looking at n-particle correlations and is the [large $n$ ] limit of the cumulants.

Tom: Okay, we can leave this for the discussion period.

Note added (Tom): The question what the Lee-Yang zeros method implies in terms of a minimum number of particles involved remains unresolved.

Edward: You have also shown that there are $\mathrm{v}_{2}$ correlations with the forward calorimeter which is way out of the range of the minijets. So you cannot say it is jets. All particles know about this direction [event plane].

Raimond: That is right, and the magnitude [of $\mathrm{v}_{2}$ ] is also the same.

Tom: That is a different question. I am asking within a given rapidity region how many particles are involved. I am not asking about identifying the reaction plane.

Raimond: But even if the average cluster size is 10 to 15 then you would see a very clear scaling in going from $n=4$ and up.

Tom: I am not arguing about minijets. 
Raimond: But this shows that the cluster size must be much, much bigger. If you measure that $\mathrm{v}_{2}\{8\}$ is not zero it doesn't mean that $\mathrm{v}_{2}\{10\}$ could be zero.

Derek: I have a couple of questions about the $\mathrm{Cu}-\mathrm{Cu}$. You showed the $\mathrm{Cu}-\mathrm{Cu}$ and after you took out the fluctuations you got something which made sense but that was some time ago and there have been improvements in understanding non-Gaussian fluctuations and also $v_{2}\{4\}$. I believe the previous results were two-particle correlation data. If you go back there now with this understanding of fluctuations would you expect the $\mathrm{Cu}-\mathrm{Cu}$ to be reduced or to remain the same? This is important.

Raimond: I haven't seen that [eccentricity] scaling done any more recently than that. At some point you cannot be too quantitative anymore. It is clear that the scaling with the standard eccentricity is really off because you get a dependence which is really unphysical. There is no way you could explain that $\left[N_{\text {part }}\right.$ dependence]. And the other scaling brings them close to each other and there are many models which predict that it [ $\mathrm{v}_{2} /$ eccentricity] would go in the same trends. Hydro models would certainly allow you to have some differences between $\mathrm{Cu}-\mathrm{Cu}$ and $\mathrm{Au}-\mathrm{Au}$ there. But I think we are trying to do too much with these Glauber models in my opinion.

Mike: If you are really interested we could have more $\mathrm{Cu}-\mathrm{Cu}$ data.

Yuri: In the region of eccentricities where you have large $\mathrm{v}_{2}$ what is the average number of collisions per participant?

Mike: That is $v$ which is never more than 6. Probably 3 .

Yuri: For $\mathrm{v}_{2}$ you have the almond, so the number of collisions decreases from the limit. How many collisions for each guy?

Note added (Tom): The maximum number of Glauber N-N collisions for a given participant nucleon is about 12 in central Au-Au. The mean is 6 . The minimum value is by definition 1 . For non-central collisions the maximum and mean are lower.

Jiangyong: He is asking about how many collisions in the final state, right?

Raimond: That is what I thought but that is not what he is asking.

Jiangyong: In a transport model you get 20 to 30 collisions.

Mike: No he is asking how many collisions per participant and that can't be more than 6 .

David: I have a comment about the eccentricity scaling in $\mathrm{Cu}$. You stated that it is unphysical. But I think it is not only physical but is totally expected because that data is event plane $\mathrm{v}_{2}$ and if there is any non-flow [jet contribution] left then the standard optical eccentricity goes to zero for most central collisions so it has to have that behavior.

Raimond: You are right. You have to be very careful that you subtract non-flow in a correct way. And in a small system like $\mathrm{Cu}-\mathrm{Cu}$ that is a lot more difficult. But this was PHOBOS data and they did the calculations of two-particles with a huge rapidity gap and they claim that they suppress all the non-flow in that way. And while you cannot prove that, it looks very probable. It is very much using the long range correlations.

Mike: I have a question about jet correlations. We trigger on a particle and look at the other and see a big $\mathrm{v}_{2}$. What $\mathrm{v}_{2}$ do we subtract?

Raimond: I don't have the answer [laughter]. 


\section{Rene Bellwied}

Christina: But if you only take our particles that we measure - protons, kaons and pions, it [statistical model fits to particle yield ratios from $\mathrm{e}^{+}-\mathrm{e}^{-}$] is kind of okay isn't it?

Rene: Several particle yields do not agree well with the model.

Raimond: It doesn't do well in terms of $\sigma$ [errors for the LEP data], right? How small are the error bars on these [LEP] points compared to how well we measure them in A-A, because they look actually quite [small?]?

Rene: These are particle yields, not ratios. There are factors of 2 differences for certain particles. The argument that has been made is that the suite of particles that you need to measure in order to make a statement like that should be as big as possible. This is the most complete fit you can find to the $\mathrm{e}^{+}-\mathrm{e}^{-}$data.

Tom: This is one side of a battle, right? And the other side is Satz - Beccatini who say quite definitively that the fit quality in $\mathrm{e}^{+}-\mathrm{e}^{-}$is exactly the same as in A-A. You really need to have the next slide with that side.

Rene: Sorry, I am biased [laughs].

Helen: I do not think Beccatini says that anymore. I think Beccatini said that back when SPS data was the best data we had.

Tom: No, this is very recent. Satz has gotten involved and this is a joint statement from the two of them within the past year.

Note added (Tom): The relevant paper is 0911.3026. The abstract states "When these requirements [comparable errors and hadron species] are maintained, the quality of the statistical model description is found to be the same for the different initial collision configurations" refering to $\mathrm{p}-\mathrm{p}, \mathrm{e}^{+}-\mathrm{e}^{-}$ and A-A.

Helen: Within the past year I have also heard him say that p-p does not fit as well as A-A.

Tom: p-p I know nothing about.

Rene: It might work all right in $\mathrm{e}^{+}-\mathrm{e}^{-}$but what would that mean?

Yuri: What about p-A? There is old NA49 data where they were claiming that the $\mathrm{K} / \pi$ ratio was increasing [with number of collisions] and that has nothing to do with equilibrium.

Rene: We have p-A data on this plot but it doesn't increase by a factor of ten. We have a phase space problem that goes away when we increase the system size and that will happen between p-p and d-A already. But then on top of this you have enhancement of strange particle production and strange hadron production that cannot be going on in hadronic interactions.

Yuri: But in p-A it wasn't, as far as I remember, a question of threshold effects as they were measuring at a given energy as a function of number of collisions. They were measuring the $\mathrm{K} / \pi$ ratio as a function of the number of collisions and they saw it increase by a factor of two.

Rene: I don't think the kaon yield is a good indicator of what's happening partonically because you can make effectively a kaon out of pion interactions in one step hadronically. You cannot make an $\Omega$ or a $\Xi$ out of a pion interaction in one step, and the time argument plays in. The yields that 
we measure in the multi-strange baryons are not achievable if you assume the system is purely hadronic. So it has to be partonic and it has to be thermalized in order for the strange quarks in the phase space to see each other and recombine into a $\Lambda, \Xi$ or $\Omega$.

Yuri: Let me understand the argument. I do not see any reason why it should be thermalized.

Rene: Right now I am talking about chemical equilibration.

Yuri: You break the vacuum with more force and you get more strange particles. There is a much more simple explanation than [the one you have described].

Mike: Okay, this is for the discussion later.

Tom: A technical point - these [blast wave] fits are commonly applied below $2 \mathrm{GeV} / \mathrm{c}$ and many of the early publications were [even] below $1 \mathrm{GeV} / \mathrm{c}$. So this is a functional form describing data over a very restricted $p_{t}$ interval, and the functional form contains three numbers: offset, slope and curvature - that's it. So you are fitting data with a function that has three parameters, and you are then interpreting them in terms of a physical model.

Edward: So these are $99 \%$ of the particles and they are associated with an explosion and $1 \%$ is not. Whatever the $1 \%$ does is a separate issue.

Note added (Tom): That is an assumption basic to hydro, not an observed fact. We observe that $30 \%$ of particles in central Au-Au are jet correlated, not 1\%. The survival of such correlations strongly challenges the assumption of a monolithic "explosion."

Jiangyong[?]: So what do you mean by a correlation length [in the Tsallis distributiuon]?

Rene: The q-parameter in Tsallis measures how far the [constituents in the] system can interact with each other which is how I would interpret correlation length, and it is actually small.

Helen: In your paradigm (4) does high $p_{t}$ mean above $2 \mathrm{GeV} / \mathrm{c}$ ?

Rene: Yes, above $2 \mathrm{GeV} / \mathrm{c}$. What I am saying is that there is a part of the fragmentation domain that is dominated by recombination.

Mike: I thought [the color transparency model] was a good explanation of the $2-3 \mathrm{GeV}$ effect [baryon/meson ratio] rather than recombination.

Rene: In recombination the question is whether or not the kaons and pions are the same. Here I am making a mass argument.

Thorsten: If it is a mass argument what about the data which show the $\eta$ [meson] is double the $\phi$ [meson]?

Rene: Right, I think what we are trying to see is whether a resonance state behaves differently than a non-resonant state. Because it [resonance state] forms and decays in the medium and if chirally symmetric may have a much shorter lifetime.

Lijuan: But the $\rho$-meson is the same as pions.

Rene: Right, and that speaks against this [model]. But again the $\rho$ is a resonance and we have to see if resonances follow different principles.

Edward: The main question [about Gavin's hydro model of the same-side ridge formation] is that they boost it and get this picture which is nice. However, you have to assume that nothing happened 
to the object, [that] it remains small. I don't understand why the objects don't expand.

Rene: A color glass condensate is quite different from color ropes in a string picture. Qualitatively they give the same picture, [but] you have to look at this quantitatively. There are many more flux tubes in a color glass condensate than strings in an initial condition in a nucleus.

Boris: Actually, it is not a fair comparison between p-p [yields] at the LHC with RHIC Cu-Cu because at the LHC the multiplicity is high because it is distributed over a huge rapidity interval. You should compare $\mathrm{dN} / \mathrm{dy}$.

Mike: But you can do this at midrapidity.

Boris: Yes you can do this and then $\mathrm{Cu}-\mathrm{Cu}$ is much bigger.

Rene: At the ALICE detector at the LHC in plus/minus one unit of rapidity, for 60 thousand events (these are in the tail of the distribution), it [the total multiplicity???] is exactly the same as for RHIC Cu-Cu. This is the tail not the mean of the LHC p-p distribution.

Che-Ming: I am still puzzled about the figure which shows that heavier quarks fragment earlier in the medium rather than in free space. In the medium, with QGP around, how can it fragment into hadrons? Does the charm quark fragment into charmed mesons in the medium?

Rene: Do you mean the picture where you make the meson then dissolve it?

Che-Ming: Yes, that is the way to suppress the D-meson production, by dissociation in the QGP. The D-meson cannot be produced in the QGP, right?

Rene: Well from the formation time argument it can be produced. But you are saying you dissociate it. It is the same argument. I think there is a difference whether you look at a heavy quark hadron or a light quark hadron. I think you can show, for instance in the paper that Anne [Sickles] has written with Stan Brodsky, that if the proton is produced early you will have a point like object and that point-like object will have a reduced cross section with the colored medium and it can make it out.

Yuri: I have a question about how you estimate this formation time. For example, for a D-meson do you really take energy divided by $\mathrm{m}$-squared?

Rene: No, what I do is that I have two-components, the boost and the energy conservation. I take the fractional momentum of the fragmentation process and I scale the time back with the fraction of momentum. So if I am required to make a high $p_{t}$ particle that high $p_{t}$ particle, because of energy conservation, has to come from quark fragmentation very early because Bremsstrahlung would not allow me to get that much energy into the hadron. So I have the uncertainty principle in one direction and energy conservation in the other direction and I have a convolution formula which gives the results.

Mike: Question for you [Yuri Dokshitzer]. Your textbook was quoted as saying that the formation time was $\mathrm{ER}^{2}$ instead of $E / \mathrm{M}^{2}$ and I think we agreed it is gamma times $\mathrm{R}$.

Yuri: For a light object yes, but for a heavy [object] it is R times gamma. You profit from producing [the hadron] very early.

Rene: It [heavy hadron] is made in the medium. The question is can it survive the medium? And the question is whether color transparency can be applied to particles that are not directly produced 
but that are produced in the fragmentation.

Boris: Concerning color transparency one should not forget why NE18 experiment at SLAC failed to see color transparency. It is because they did produce a small object but it is expanding too fast. For the same reason a proton in the medium, whatever it is, is expanding at this $p_{t}$ very fast, you will never see any color transparency.

Rene: Well the problem is that the fragmentation process does not move at the same speed as the plasma. So the question is how long does it [proton???] really stay in the medium, because if you believe the models the plasma expands at $60-65 \%$ the speed of light and the fragmentation is quite a bit faster. So what I am trying to say is that if it is formed in the medium it might make it out of the medium because of the decoupling of the medium and the fragmentation.

Edward: A comment to this point. We discussed that heavy final particles are not produced outside the medium. I want to emphasize the other part of the curves which say the pions are produced outside the medium. This is very important. It is important for Tom [Trainor] because his fragmentation does not interfere with hydro because they are outside already and produced way outside the medium. But that will also be important for Rudy [Hwa] because he considered coalescence of hard and soft and I am saying that hard and soft are spatially separated. They might have the same small momentum, the guys that came from hard and soft origins, but they are spatially separated.

Rene: Then let me make an argument in Tom's defense. This has a fragmentation cut-off. We only put this [pion formation time] curve down to $2 \mathrm{GeV} / \mathrm{c}$. If you believe that fragmentation can actually populate the soft bulk then that soft bulk probably would sit in the medium.

Edward: But we do not discuss below 2, we are discussing 2 to $6[\mathrm{GeV} / \mathrm{c}]$ where there is or is not coalescence. The difficulty with coalescence between those two components is that they are spatially separated. One is moving at the speed of light and the other is moving at the speed of hydro.

Rudy: But it takes time for the hard parton to fragment into the shower parton, which is different than the hard parton.

Thorsten: I don't think there is an issue of whether fragmentation processes can populate below 2 $\mathrm{GeV} / \mathrm{c}$, I think they can. The question is how large is this component and if it happens inside the medium because it is low $p_{t}$ and the formation time gets shortened does it not get thermalized and become a part of the medium? So the question is not if fragmentation can produce particles at 2 $\mathrm{GeV} / \mathrm{c}$. The question is how large is the fraction of hadrons produced by fragmentation below 2 $\mathrm{GeV} / \mathrm{c}$ which gets out.

Rene: Right.

Jiangyong: If you have this color transparency there is no interaction. For example, as a proton goes through the medium there is no strong interaction. Why in a proton + heavy ion collision is there any interaction?

Edward: This is what Yuri [Dokshitzer] was saying. There is a big difference between two protons colliding, [each] with a big cloud around them and these guys [prehadrons formed in the medium] which are produced naked.

Rene: That is correct. In color transparency the proton is point-like whereas in p-A the proton is a 
fully formed object. So you could say that these are not real protons and that is what I was saying about pre-hadron formation. It has its quark constituents and is color neutral but is just not fully dressed yet.

\section{Che-Ming Ko}

Yuri: What do you mean by string melting?

Che-Ming: Strings produced from the initial collision normally fragment into hadrons. We don't

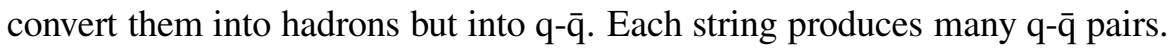

Yuri: How do they [Fochler, Xu, Greiner] treat 2-to-3 processes?

Che-Ming: They used the Gunion-Bertsch formula to correct for the LPM effect. It is a simple formula to model this effect.

Guy: They [Fochler et al.] are doing something very suspicious. When they set the strong coupling to 0.001 and calculate the shear viscosity their answer is reduced a factor of five by these 2-to-3 processes. We do a leading order calculation, which should work when $\alpha_{s}$ is 0.001 [???] and find that it is reduced about $20 \%$. So they are doing something grossly wrong in their 2-to-3 processes.

Edward: But even if they are doing it right there are 2-to-4 processes and the whole series is divergent.

Derek: The problem I think is that their co-linear emission is not at all co-linear because they set a typical angle by some Debye scale and it ends up being infinity, so two guys come in and a third guy goes off at some random direction.

Rene: Do you need the 2 -to-3 processes [to fit the proton/ $\pi$ ratios]?

Che-Ming: We do not need $2 \rightarrow 3$ processes but use a large cross section for the $2 \rightarrow 2$ process.

Thorsten: What is the dynamics inside the [AMPT] model [that causes the away-side double bumps]?

Che-Ming: The dynamics is parton scattering. As I said that in the AMPT model, we get hadrons from HIJING and converted them into q-q. The q-q collisions are then taken into account by parton cascade. When quarks and antiquarks stop scattering, they are converted to hadrons.

Thorsten: But which of the steps generates the structure?

Che-Ming: I don't know. I am just quoting their analysis. They use our code as a black box for generating heavy ion collision events.

Jiangyong: Does AMPT contain all the initial state participant fluctuations?

Che-Ming: Yes, everything.

Jiangyong: And we know when you run hydro with fluctuations in the initial geometry we see a double hump structure on the away-side. One way to check that is to explicitly embed a high energy jet into AMPT which is what we did and you don't see this [double hump structure]. Initial geometry fluctuations are based on the Glauber model.

Mike: Why does the initial fluctuation give you two humps? 
Jiangyong: Because they have large cross sections which mimic the hydrodynamic evolution. They produce the $\mathrm{v}_{2}$, right? So if you reduce the cross section you will not have this feature anymore.

Edward: So wait, there is a jet, right?

Che-Ming: Yes, there is a jet by triggering on a $6 \mathrm{GeV} / \mathrm{c}$ hadron.

Edward: So your model basically mimics hydro because of the large cross section.

Thorsten: What happens when you embed a jet explicitly?

Jiangyong: When you embed a di-jet at a fixed point there is no fluctuation, and if you check whether these jets produce a Mach cone we find that they don't.

Che-Ming: But their analysis is based on all particles in an event, like in experiment.

Mike: The theorist has a model that is so good that you run it and get something totally unexpected and it fits an experimental results and nobody knows why. Great, continue!

Edward: [It is] because his model is very complicated but the physics is the same [as in hydro].

Rainer: But there is a difference [from Rudy's coalescence model] in that you do not develop a shower picture and that takes time also.

Edward: There is one thing missing [in the deuteron coalescence] which is that nobody hits your deuteron. The deuteron is not absorbed; it is just a transparency model.

Che-Ming: No, that is the coalescence model but in the dynamical model we do have absorption.

Edward: That is presumably the difference between the two.

Thorsten: Does the [coalescence model] know about coordinate space?

Che-Ming: Yes.

Thorsten: Are there any plots where you do not care about coordinate space to see if that makes a difference?

Che-Ming: Yes, in a previous paper, but I do not have that in this talk.

Thorsten: Can you tell me how it looks?

Che-Ming: Those results [coalescence vs dynamic models] are much closer, but the magnitudes are different. The calculations involved only momentum space. From the proton and neutron momentum distributions, we calculated the deuteron production rate in the dynamic model but the yield in the coalescence model.

Lanny: Did the Chinese group [who???] look at di-jet events or di-jet correlations with AMPT?

Che-Ming: They wrote a paper which I found later, but they did not include it in that paper.

Rene: If you look at the baryon over meson ratio that you showed for the $\Lambda_{c}$ over D [meson], I wonder if you can apply this to any baryon over meson ratio, [for example] the proton over pion or the $\Lambda$ over kaon and whether I can exclude, from [the] coalescence [results], that I have di-quark formation? Because in a di-quark model you would always be peaked at a lower momentum, right? That is also true for the non-charm baryon. So if the curve we measure experimentally has its mean at $3 \mathrm{GeV}$ or so you could say that [this result] cannot be [from] true di-quark formation. 
Che-Ming: Yes, in the proton case di-quark formation does not seem to be important. But the point is that the proton and $\Lambda_{c}$ may not be just di-quark and quark states.

Rene: But the u-d would be the di-quark [component]?

Che-Ming: No, it depends. The $\Lambda_{c}$ is computed with many configurations and which is more important? We should ask someone working on quark models which [of the above particles] has the largest di-quark component.

Denes: You showed the calculation of the $\phi$ [meson] in AMPT, and actually you have an old one with a $\phi$ and an $\Omega$.

Che-Ming: Yes, but that one differs from the one given here where the $\phi$-meson is obtained within the AMPT code by running it from beginning to end. In the other paper of ours, the parton cascade was run to the end, and the parton coalescence is then used for $\phi$-meson production.

Denes: The main conclusions for me were two things. One, the $\phi$ versus $\Omega$ could be scaled into each other, which was nice. The big killer conclusion in your old paper was that the quark flow was very different from the rescaled $\mathrm{v}_{2} / \mathrm{n}_{q}$ versus $p_{t} / \mathrm{n}_{q}$. If you take the $\phi$ flow results from AMPT and you do the rescaling you hope to get the underlying quark flow, but you don't. Is this still true? Because that is the main thing. Experimentalists look at this and they think there is quark number scaling, so it must be coming from the quarks and there is a simple, cheap formula. But you have detailed dynamics which show that it doesn't, even if there is scaling between mesons and baryons. And when you do the rescaling divided by quark number you don't get quark scaling. It is a factor two [different]!

Che-Ming: Really!? I think we tried to fit the $\phi / \Omega$ number by adjusting the size.

Denes: But it is constrained by the final number, you do not have any room.

Lijuan: For the deuteron $v_{2}$ the transport model did a bad job compared to coalescence. When experimentalists used the deuteron spectrum and proton spectrum to get the $\mathrm{v}_{2}$ variables and [not understandable?] to get the correlation [?] size. If the transport model does a bad job does it mean we should not do this specifically or do you have any comments?

Che-Ming: Deuterons are very big objects and the internal momentum spread is pretty big, everything is big. Therefore, it is not bad as far as the scaling is concerned. I remember a paper by Yu-Gang Ma and his collaborators who claimed that in a low energy heavy ion collision deuteron and triton all scale, but he did not use the kind of coalescence used in our paper. He used another kind of coalescence by obtaining the nucleon spatial and momentum distributions from a transport model, which means that he looked at neutrons and protons in the coordinate and momentum space and played a little bit with the parameters for their coalescence to get the scaling. Here, we use the wave functions of deuterons and have no parameters. Our dynamic phase space freeze-out distribution is, however, not very well calculated. We apply a transport model to calculate the neutron and proton distributions, and I am not sure it is perfect.

Edward: The question is not whether it is perfect or not, the question is what Denes [Molnar] was saying. There are two competing ideas about why different particles have different $v_{2}$. One is the hydro idea. In other words I am not going to get a cross sectrion larger than the pions. That is why it results later [not understandable?]. That is what Derek [Teaney] will speak about. The other is the 
recombination idea which gives partonic flow and then it is to be multiplied by two or three. Since you have a detailed model you can check. What is the reason for the difference? [Is it] the cross section difference, or the time that it takes two or three quarks to somehow recombine differently or whatever. Which explanation or idea is right? You can check independently of the data.

Che-Ming: Yes, that is a good idea.

Rene: But that is not quite fair because what most models do in order to extrapolate the cross section is they apply the quark sum rule to the cross section probability. That is what RQMD is doing. If you put the quark sum rules into the cross section then you get out what you put in. If you put in 2 to 3 scaling in the cross section then you get out 2 to 3 scaling. That's not a [testable prediction].

Edward: If it comes from the cross section I understand their argument. If it comes from some mysterious multiplication of $v_{2}$ by 2 or 3 , then who knows? Hydro says there are 2 or 3 times more fluid elements flowing here than there. What does that have to do with quark scaling? I never understood that. Whether you are made of 2 quarks or 15, what's the difference? There should be the same hydro $\mathrm{v}_{2}$ for all particles.

Mike: Let's move this to the discussion but I will end this wonderful talk by telling the middle line from a joke about French engineering schools. The middle line is "The mid-level French engineering school builds the bridge, it stays up, but they don't know why." [laughter] This reminds me of AAMPT explaining everything but they don't know why.

\section{Rainer Fries}

Rene: Can I ask a question about this [hadronization mechanisms]. I think there is a distinct difference, because when you have HERWIG and a cluster hadronization model the clusters are considerably larger than the hadron. So you are actually breaking the cluster, whereas you are recombining partons into exactly the right hadrons.

Rainer: Ah, yes. In a sense maybe this is more related to a statistical hadronization model where you also can start from clusters and you sort of produce these hadrons in equilibrium. It is certainly not quite the same thing.

Tom: We should note that when we go from $p_{t}$ to $m_{t}$, which is the $\mathrm{KE}_{T}$, we are minimizing by at least a factor of three sensitivity to the boost distribution, which is nominally the object of study in $\mathrm{v}_{2}$. So this is a funny thing to do.

Ulrich: What do you mean by boost distribution?

Tom: The thing that is causing the displacement on $p_{t}$. In other words, built into your hydro model is a boost, a Hubble expansion, and that is producing the displacement. When you go from $p_{t}$ to $m_{t}$ you are reducing sensitivity to boost by at least a factor of three.

Rainer: That is exactly the point of that plot $\left[\mathrm{v}_{2} / \mathrm{n}_{q} \mathrm{vs} \mathrm{KE}_{T} / \mathrm{n}_{q}\right]$.

Ulrich: The point is that the $m_{t}$ scaling in the low kinetic energy region is characteristic of thermalization, weakly broken by flow, so weakly that on the scale shown here you cannot see it. 
Rainer: By showing that there is a universal curve, that makes a strong argument that there is hydrodynamics, or thermalization in the first place.

Mike: So Uli, you like the middle plot $\left[\mathrm{v}_{2} / \mathrm{n}_{q}\right.$ vs $\left.\mathrm{KE}_{T}\right]$ best.

Ulrich: That plot is the one I am going to use to make my point about hydrodynamics. He [Rainer] wants the right plot $\left[\mathrm{v}_{2} / \mathrm{n}_{q}\right.$ vs $\left.\mathrm{KE}_{T} / \mathrm{n}_{q}\right]$ because that carries over into recombination. And that is important because the two paradigms, in quotation marks, refer to different regions of $p_{t}$.

Rene: There is always this question about how far down in $p_{t}$ recombination applies. If I take the $p_{t}$ plot and divide it by $\mathrm{n}_{q}$ I get pretty good agreement below $2 \mathrm{GeV} / \mathrm{c}$.

Rainer: Well it is not too bad, but certainly the pions are not doing too well.

Ulrich: In $p_{t}$ the pion and the heavier-particle $\mathrm{v}_{2}$ split because of hydrodynamic flow, and you cannot undue that splitting by this scaling.

Rene: What I am trying to say is even particles that follow hydrodynamics are probably formed by recombination first.

Ulrich: What do you mean by "instantaneous" coalescence.

Rainer: I mean a fixed hyper-surface.

Guy: One thing that has always bothered me about this is that it seems like it violates the second law of thermodynamics. The first law of thermodynamics doesn't bother me because I imagine that all these things have relatively large interaction energies and the reason they are coalescing is because the energy of the baryon is lower. So in the energy there is some sort of slop if you worry about the interaction energies. But the entropy seems like a serious problem.

Che-Ming: The reason is that most are formed as resonances; most are formed as rho mesons rather than pions. So we can calculate the entropy, and it is only slightly reduced. The shortage is a few percent.

Rainer: If you just do that at high $p_{t}$ then there is no problem of entropy.

Ulrich: But that is not a real argument, because if your resonances were infinitely long lived then you would still have the same problem.

Che-Ming: The degeneracy of hadron resonances leads to the gain of a huge factor in the entropy of formed hadrons.

Edward: You are saying that you may reduce [the entropy] first, then there is another mechanism that increases it back.

Che-Ming: No, I am saying that if you push to zero $p_{t}$ then you violate entropy.

Edward: What does it have to do with $p_{t}$ ? You combine three particles into one.

Rene: There is a reservoir that takes care of the entropy.

Mike: Let's move this to the discussion time.

Yuri: Gentlemen I don't think there is any reason to worry because we are talking about a time where your definition of entropy just doesn't make sense before the final [?] state. 
Rene: Is that [thermal partons coalescing to thermal hadrons] really true? I thought the Rho and Mueller[????] paper made the argument that you can have a power law quark spectrum and get a thermal hadron spectrum.

Rainer: If you fold it often enough somehow, right?

Rene: Do the partons have to be thermal in order to get a thermal hadron spectrum?

Rainer: I don't think that this is a requirement. Certainly you cannot do it with wave functions that are very narrow because then you will just go back into a power-law [spectrum].

Guy: If this [Boltzmann transport] model has detailed balance why do you have hadrons and not still have some mix of quarks?

Rainer: There are still quarks. At least [in] so far [as] the quarks are treated basically as a reservoir. Guy: So you don't complete hadronization.

Rainer: No, it is not an exclusive hadronization model where you try to convert all the quarks and anti-quarks into hadrons. You just ask the question what are the kinematic properties - the flow and the spectrum or the $v_{2}$. There is no mechanism in there now where the quark distribution is also dynamic.

Denes: Does the heat bath stay constant? There is no reaction back on the heat bath [is there]?

Rainer: No, mesons can also decay back into quarks and anti-quarks. There is no dynamical evolution equation for the quark or anti-quark distributions.

Denes: In some sense nothing is conserved, not the energy, not the momentum [laughter].

Rainer: No. Microscopically, energy and momentum are conserved. I don't deplete the heat bath.

Denes: So you would not notice if it is not conserved either.

Rainer: I don't understand.

Thorsten: It [the heat bath] is infinite. That is why it is conserved.

Rainer: Microscopically, energy-momentum is conserved in the process.

Denes: But then your heat will flow differently.

Rainer: The heat bath is just sitting there.

Denes: So that is why it is not conserved.

Rainer: The question you can ask is if you have a certain flow pattern in your heat bath how does it imprint onto the [not understandable?] I said before.

Denes: But you have to assume the pattern stays as a function of time because you are running it in time to describe the evolution of the heat bath. You are solving [the] Boltzmann [equation] so there must be time [dependence].

Rainer: No, we only look at the equilibrium limit. You could ask how long this takes. But that is not addressed in this study. You can ask what the kinematic properties are of the particles you produce.

Denes: Okay, I see. 
Edward: But how do you decide at which temperature it happens? If you never consider a probability for this particle to escape it can be produced earlier or later. You just arbitrarily decided to do it at some place [?].

Rainer: No, there is nothing else in this equilibrium distribution of mesons. So in principle you can think of them in a box because you have stopped your evolution at that point. So there is no time evolution of the fireball.

Tom: You have $\mathrm{T}_{c}$ here which is maybe $2 / 3$ of $\Lambda_{Q C D}$. So what means "scattering" in the partonic system. You heard from Yuri [Dokshitzer] that we have angular ordering due to quantum mechanical interference.

Rainer: There are no perturbative quarks or gluons [here]. The mesons are basically just BreitWigner shaped resonances. That means quarks and anti-quarks don't scatter through an angle, they scatter into this [resonance] state.

Tom: When $\mathrm{T}_{c}$ is $\Lambda_{Q C D}$, what means scattering?

Rainer: The only process is $\mathrm{q}+\overline{\mathrm{q}}$ going to $\mathrm{M}$ going back to $\mathrm{q}+\overline{\mathrm{q}}$. There is no perturbative QCD here.

Boris: I just wanted to comment that there is no problem with energy conservation in coalescence because you never produce a hadron of certain mass as in fragmentation. You produce a bare hadron which doesn't have any mass. It needs formation time to develop mass. This uncertainty principle, you cannot break it.

Rainer: Yes, but I think it is a sort of dual description from pre-hadron formation to this [recombination] because here the quark would already have constituent quark mass. So these descriptions might be dual in some way.

Rene: This is important, because if this [recombination] would go via massless pre-hadrons it would be a completely different picture than what he [Rainer] described. What he [Boris] described was having colored objects recombine into colorless objects.

Boris: But these objects don't have mass.

Rene: But they have constituent quark mass.

Boris: Well, quarks may have mass, but they do not fuse into a certain hadron mass.

Rainer: Do you [Boris] also expect this picture for the bulk of the hadron production? If you really hadronize your quark-gluon plasma with current quark masses for quarks, then you first form prehadrons, then after a certain formation time you have full hadrons.

Mike: I can answer that question. An exponential distribution of quarks is a quark-gluon plasma. If you believe in a quark-gluon plasma it has to apply to everything, not just hard scattering. So you figure it out.

Thorsten: Rainer, can you briefly comment on the point Edward [Shuryak] brought up earlier about the intermediate region where something off-equilibrium might recombine with something from the equilibrium bulk? How good is the space-time picture of this process under control? Is this really an issue that the hard part might be out of the medium by the time you do the recombination?

Rainer: I think there is a problem with different time scales. So we never had a soft-hard recombination in our model because they do live on different time scales. The medium is evolving and 
the jet is going out of your medium There are probably mechanisms where you can probably pick something up out of the medium, but it might be suppressed because of the space-time evolution. I think it is a complicated problem. 